\title{
The influence of object-image velocity change on perceived heading in minimal environments
}

\author{
CHRISTOPHER J. BEST \\ Deakin University, Melbourne, Victoria, Australia \\ ROSS H. DAY \\ La Trobe University, Melbourne, Victoria, Australia \\ and \\ BORIS CRASSINI \\ Deakin University, Melbourne, Victoria, Australia
}

\begin{abstract}
When human observers move forward and rotate their eyes, a complex pattern of light flows across the retina. This pattern is referred to as retinal flow. A model has been proposed to explain how humans perceive their direction of self-movement (or heading) from (1) static depth, (2) direction of image motion, and (3) whether image velocity undergoes acceleration or deceleration (Wang \& Cutting, 1999). However, findings from past research in which sparse or minimalist stimuli were used have suggested that not all of the information to which participants are sensitive is captured within the scope of this model. In particular it has been suggested that the magnitude or size of image velocity change may be of significance beyond simply whether image velocity could be categorized as speeding up (i.e., accelerating) or slowing down (i.e., decelerating). In two experiments, the influence of this factor on heading judgments under minimal conditions was investigated. Evidence was found in support of the idea that the rate of image velocity change can influence judgments of the direction of self-movement in minimalist conditions.
\end{abstract}

During navigation through the environment, the structure of light reflected from surfaces to the observer's point of view changes. Gibson (e.g., 1950) called this change motion perspective. He proposed that motion perspective is involved in the perception of both the layout of the environment in depth and the observer's movement through it. According to Gibson, the critical stimulus correlate for perception of movement direction (or heading ${ }^{1}$ ) is the focus of expansion (FOE) - that is, the point from which all other points appear to radiate during movement. Since Regan and Beverley (1982) demonstrated that the FOE could not specify heading in the presence of eye rotations, numerous other models have been presented to explain how it is that humans perceive their direction of heading. Many models have shared fundamental characteristics, including a focus on (1) global visual motion as the primary source of information for heading perception and (2) the direction of heading in retinotopic terms. An alternative approach is to consider the direction of heading relative to objects in cluttered naturalistic environments. This has been the focus of the model developed by Cutting and colleagues (e.g., Cutting, 1986, 1996; Cutting, Alliprandini,

Correspondence concerning this article should be addressed to C. J. Best, School of Psychology, Deakin University, Burwood Campus, 221 Burwood Highway, Burwood, VIC, 3125 Australia (e-mail: cjbest@deakin.edu.au).
Creutz, \& Wang, 1998; Cutting, Springer, Braren, \& Johnson, 1992; Cutting, Vishton, Flückiger, B aumberger, \& Gerndt, 1997; Cutting, Wang, Flückiger, \& Baumberger, 1999; Vishton \& Cutting, 1995; Vishton, Nijhawan, \& Cutting, 1994; Wang \& Cutting, 1999). According to this model, perception of heading is based not on global motion, but rather on (1) categories of local relative motion that occur between the retinal images of objects during self-movement and (2) information about the layout of objects in depth, including static depth information.

\section{The Object-Based Model of Heading Perception}

A most recent version of the so-called object-based approach $^{2}$ was set out by Wang and Cutting (1999). They argued that three categories of relative motion describe all relative motion between objects in the field of view during forward self-movement. In this situation, object images move closer together with accelerating velocity (convergence; $\mathrm{CV}$ ), farther apart with accelerating velocity (accelerating divergence; AD), or farther apart with decelerating velocity (decelerating divergence; $\mathrm{DD}$ ).

These three categories of relative motion occur only between objects in particular locations in the environment with respect to the observer's path (see Figure 1). Therefore, each category of relative motion imposes some constraint on the possible direction of heading in terms of the regions of the visual field around a pair of objects. 


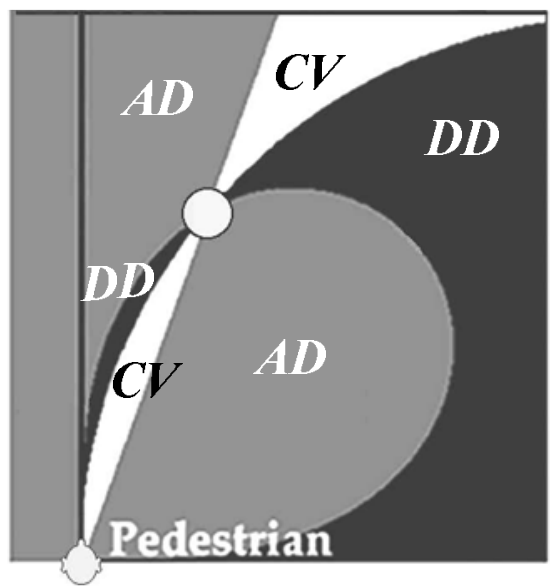

Figure 1. Snapshot of retinal image relative motions. Retinal image motions are shown for objects around a fixated reference object. An observer moving directly up the page, looking off to the right, is depicted. The relative motion between all pairs of object images in retinal flow can be described in three ways. Convergence $(\mathrm{CV})$ refers to reduction in the angular distance between the retinal images, accelerating divergence (AD) refers to an increase in that distance with increasing velocity, and decelerating divergence (DD) refers to an increase in that distance with decreasing velocity. From "Where We Go With a Little, Good Information," by R. F. Wang and J. E. Cutting, 1999, Psychological Science, 10, p. 71-75. Copyright 1999 by American Psychological Society. Adapted with permission.

When CV or DD occurs between two object images, the direction of heading is always toward the region outside the member of the pair nearest the observer (see the left and middle panels of Figure 2). When AD occurs, the likely direction of heading can be described in terms of a probability distribution: Heading is to the outside of the object farthest from the observer $69 \%$ of the time, between the two objects $23 \%$ of the time, and outside the object nearest the observer $9 \%$ of the time (see the right panel of Figure 2). In summary, two of the sources described by Wang and Cutting (1999) — namely, CV and DD—provide invariant heading information (indicating that heading is definitely toward the outside of the near member of the pair), whereas one, $\mathrm{AD}$, provides heuristic heading information (indicating that heading is most likely to be toward the outside of the far member of the pair).

Although Wang and Cutting (1999) presented evidence to support this model, findings from more recent experiments have suggested that the relationship between object-image relative motion and perceived heading may be more complex. In recent experiments involving simulations of self-movement through minimally populated environments (Best, Crassini, \& Day, 2002; Cutting \& Wang, 2000), Wang and Cutting's model has been investigated in its simplest form: relative motion between a single pair of objects. The advantage of investigating the object-based model in this way is that information sufficient for object-based heading perception to occur is available, whereas that from global motion is minimal.

In their experiments on heading in minimal environments, Cutting and Wang (2000) found that judgments generally followed the expected pattern (see Figure 2). However, the degree of angular separation between object images and the rate at which they accelerated apart were also found to contribute to heading judgments when pairs diverged and accelerated (i.e., AD). These two factors interacted so that acceleration was a particularly strong determinant of heading judgments when object images were close together. In regard to the invariants, DD and CV, Cutting and Wang's experiments confirmed earlier findings that DD was a relatively weak determinant of heading perception and CV a relatively strong determinant (e.g., Cutting et al., 1999). Similarly, the findings reported by Best et al. (2002) supported the predictions of the object-based model. However, it was also found that the categories of relative motion described by Wang and Cutting (1999) did not account for all of the information influencing judgments of heading. In conditions in which fixation was simulated on a far reference object (RO), a near second object (SO) could be placed in two large areas of the environment in which objects underwent AD (see Figure 1). By placing the SO in these areas, different path conditions that gave rise to
Probability of Heading Direction

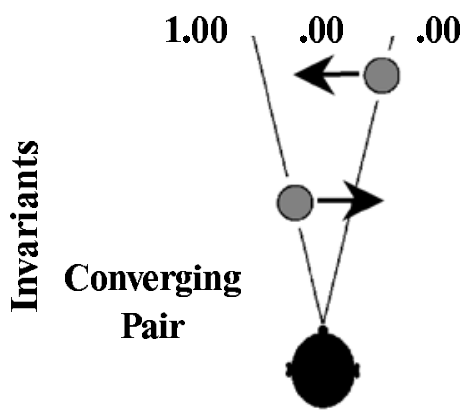

Probability of Heading Direction

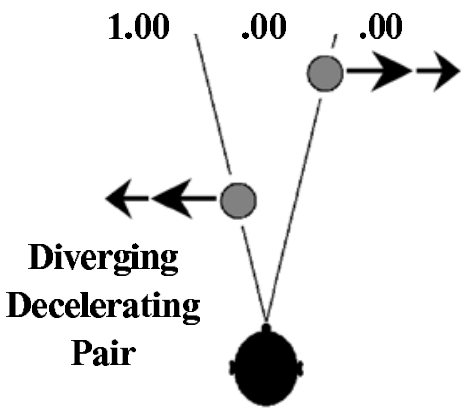

Probability of Heading Direction

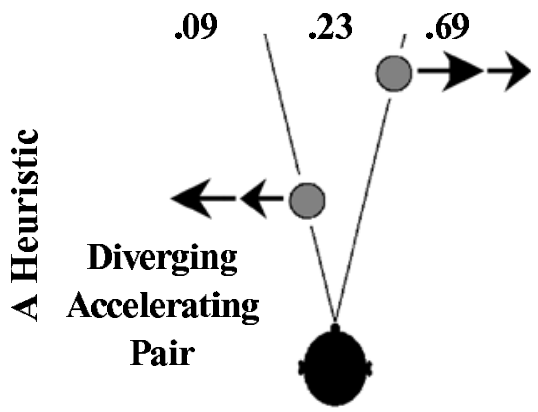

Figure 2. Wang and Cutting's (1999) description of the object-based heading information available in retinal flow. Two kinds of relative motion, $\mathrm{CV}$ and DD, provide invariant information. The third, AD, provides only probabilistic information. From “Where We Go With a Little, Good Information,” by R. F. Wang and J. E. Cutting, 1999, Psychological Science, 10, p. 71-75. Copyright 1999 by American Psychological Society. Adapted with permission. 
the same kind of relative motion (i.e., AD) were generated. The two simulated paths giving rise to AD were (1) paths between the two objects in the display and (2) paths outside the far object. It was expected from the predictions of the object-based model that heading judgments in these conditions would be identical, given that the same relative motion category was available in both conditions. However, a repeated finding was that responses differed.

In other conditions, in which fixation was simulated on a near RO, the far SO could be placed in large areas of the environment giving rise to invariant information (i.e., DD and CV). Response differences were also found between conditions containing different invariants. When the SO was placed so that DD was the only invariant in the displays, performance was poor. However, when the SO was placed so that DD and CV both occurred at different times during the simulated self-movement (a situation referred to as "multiple temporal constraint;" Best et al., 2002, p. 898), performance was accurate and in accord with predictions (see Figure 2). It is possible that this performance improvement was due to the presence of multiple invariant sources of information over time, which served to provide greater certainty about the direction of heading. However, it is also possible that DD is a weak determinant of heading perception, despite its putative status as an invariant in the object-based model.

\section{General Rationale and Aims}

The findings that participants (1) differentiated between heuristic (AD) paths in far-RO conditions and (2) did not judge heading equally well in all near-RO-invariant (DD and $\mathrm{CV}$ ) conditions suggested that even in the very simple case of navigation through a two-object environment, not all of the information that influences heading perception is captured in Wang and Cutting's (1999) threecategory object-based model. The purpose of the two experiments reported here was to identify the sources of information that appear to influence heading perception in minimalist object-based displays.

\section{GENERAL METHOD}

The methods used to generate stimulus displays, present these to participants, and collect responses were the same across the two experiments that are reported. These general methods are described in this section. Only those manipulations unique to each experiment are reported in later Method sections.

\section{Stimulus Displays and Experimental Task}

Two-object stimulus displays were presented on a computer monitor with screen dimensions of $300 \mathrm{~mm}$ horizontal $\times 225 \mathrm{~mm}$ vertical, which was connected to a Pentium II based PC. Stimuli were drawn at a screen resolution of $800 \times 600$ pixels, using a commercial three-dimensional graphics engine (MindRender by ThemeKit Systems, Leicester, U.K.). The displays were similar to those used by Best et al. (2002). Movement of an observer relative to the two objects was simulated. Each trial consisted of a period of simulated forward movement, combined with rotation relative to the two objects, with fixation on the RO. The simulated rotation served to hold the RO in the center of the field of view, while the image of the SO moved laterally across the screen. The images of both objects loomed as the observer's simulated position approached. The two objects in these displays were vertical poles with rounded ends and unshaded, untextured surfaces. The absence of self-occluding edges, shading, and texturing on the objects served to eliminate any apparent rotation of the objects, which could potentially have contributed to heading perception (Beusmans, 1998; Wallach, Stanton, $\&$ Becker, 1974). The RO was always yellow, and the SO was white.

The objects appeared to be standing on an invisible horizontal surface in an otherwise empty black virtual space. In all conditions, the RO was in the center of the field of view. In some conditions, the SO was on the left of the RO, and in others on the right. In some conditions, the SO appeared to be behind the RO, and in others in front. As is shown in Figure 1, the relative motion between the images of two objects during self-movement is dependent on the positions of the objects relative to one another and on the observer's path. This was useful in the experiments, since different kinds of object-image relative motion were simulated by placing objects in different positions in the virtual environment. A number of final gaze angles, defined as the angular separation between path direction and the observer's line of sight to the RO at the end of the simulated movement, were used. The smallest gaze angle was $2^{\circ}$ and the largest was $8^{\circ}$.

All measurements of size and distance in the virtual environments were scaled for an observer with an eye height of $1.6 \mathrm{~m}$. The objects were $3.2 \mathrm{~m}$ tall, and self-movement was simulated at a speed of $2 \mathrm{~m} / \mathrm{sec}$. Cutting et al. (1992) estimated that the distance required by a walker to avoid collision with an obstacle at a speed of $2 \mathrm{~m} / \mathrm{sec}$ is $7.8 \mathrm{~m}$. The simulations used in the experiments reported here involved final simulated distances of $10 \mathrm{~m}$ (Experiment 1) and $8 \mathrm{~m}$ (Experiment 2) from the RO.

\section{Participants and Procedure}

Male and female participants, between 17 and 49 years of age, were tested individually. Six male and 4 female participants took part in Experiment 1. Eight male and 2 female participants took part in Experiment 2. Four participants took part in both experiments. All had participated earlier in similar experiments but were naive as to the hypotheses being tested. Testing took place in a laboratory that was illuminated only by the monitor. The participants were seated directly in front of the monitor and, although unconstrained, were encouraged to maintain a viewing distance of about $40 \mathrm{~cm}$. Their task was to observe the computer-generated displays and to indicate at the end of each trial, by pressing one of three buttons, whether the simulated direction of movement appeared to be (1) toward the left of the left object in the display, (2) between the two objects, or (3) toward the right of the right object. This nominal heading task served to test predictions from the object-based model (see Figure 2). The participants were instructed to fixate the RO during the trials. Fixation was not monitored. The participants were not provided with feedback as to the accuracy of their responses. A trial consisted of the following sequence of events. The first frame of the simulation appeared and remained stationary for a brief time. This was followed by a period of simulated self-movement, after which the final frame remained on the screen while the participants made their responses. The participants were instructed to respond only when the motion in the display had ended.

\section{EXPERIMENT 1 Accelerating Divergence: A Heading Heuristic}

Wang and Cutting (1999) claimed that the three categories of relative motion depicted in Figures 1 and 2, in combination with depth information, accounted for judgments of the direction of self-movement relative to objects. However, Best et al. (2002) found that patterns 
of responses differed between different path conditions that gave rise to the same relative motion category. This suggested that the responses were based on other sources of information. It was noted that two large areas of the visual field in front of the far RO contained SOs that underwent AD during simulated self-movement. When the $\mathrm{SO}$ was placed outside the path of movement (referred to as far-RO, path-between conditions; see $\mathrm{SO}_{\mathrm{A}}$ in Figure 3 ), the participants indicated consistently that they perceived the direction of heading to be between the objects more often than when the SO was placed in front of the RO and on the opposite side of the RO to the path of movement (referred to as far-RO, path-outside-far conditions; see $\mathrm{SO}_{\mathrm{B}}$ in Figure 3). Thus, the pattern of responses in these two conditions was different despite the fact that both displays depicted the images of the object pair as diverging with accelerating velocity (AD).

By definition, the images of objects in retinal flow undergoing $\mathrm{AD}$ move progressively farther apart, and their relative velocity increases over time. Because of the acceleration of this relative motion, the farther apart objects in areas of AD become, the faster they move. Thus, in the configurations simulated in the experiments by Best et al. (2002), SOs undergoing AD necessarily traveled faster as the lateral separation between the images of the $\mathrm{RO}$ and the $\mathrm{SO}$ grew. Although objects underwent this kind of relative motion in both kinds of heuristic conditions (i.e., path between and path outside far), there were identifiable differences between the displays from these conditions.

The path conditions in question were generated by positioning the $\mathrm{SO}$ in two different areas of $\mathrm{AD}$ in retinal flow (see Figure 3). One area was on the opposite side of the RO to the path of motion (i.e., for paths outside the $\mathrm{RO}$ ), and the other was on the opposite side of the path of motion to the RO (i.e., for paths between the objects). The relative retinal image velocity of three objects (RO, $\mathrm{SO}_{\mathrm{A}}$, and $\mathrm{SO}_{\mathrm{B}}$ ) during simulated approach is represented in Figure 3. In the left panel, the simulated path of movement is shown between the $\mathrm{RO}$ and $\mathrm{SO}_{\mathrm{A}}$; the path of movement is outside the far member of the pair made up of the $\mathrm{RO}$ and $\mathrm{SO}_{\mathrm{B}}$. In the right panel, the relative velocity of objects over the course of the displays is shown as a velocity-time graph. The upward slope of both curves in the right panel is representative of accelerating, diverging motion - that is, AD. The two object pairs in this configuration (i.e., $\mathrm{RO}-\mathrm{SO}_{\mathrm{A}}$ and $\mathrm{RO}-\mathrm{SO}_{\mathrm{B}}$ ) are matched for final lateral separation in the display. It can be seen from panel B that as the observer approaches the configuration, both pairs of object images give rise to AD. However, there is a clear difference. The image of the SO accelerates more rapidly for paths outside the RO (i.e., considering the object pair $\mathrm{RO}-\mathrm{SO}_{\mathrm{B}}$ ) than it does for paths between the objects (i.e., considering the object pair $\left.\mathrm{RO}-\mathrm{SO}_{\mathrm{A}}\right)$.

Schmerler (1976) has argued that the variable that determines the salience of perceived accelerated motion is the ratio of initial to final velocity over a given time or distance of travel. This characterization of acceleration will be referred to as the velocity ratio. In the example shown in Figure 3, the velocity ratios are 1:3.94 and $1: 3.21$, indicating greater acceleration took place for the path outside the far object (i.e., $\mathrm{RO}-\mathrm{SO}_{\mathrm{A}}$ ) than for the path between the objects (i.e., $\mathrm{RO}-\mathrm{SO}_{\mathrm{B}}$ ). Another way to conceptualize velocity change is in terms of units of velocity over units of time-for example, degrees of visual angle per second per second $\left(\mathrm{deg} / \mathrm{sec}^{2}\right)$. This will be referred to as the rate of acceleration. The latter measure also reflects the greater velocity change for the path outside the far object than for that between the objects. The
A

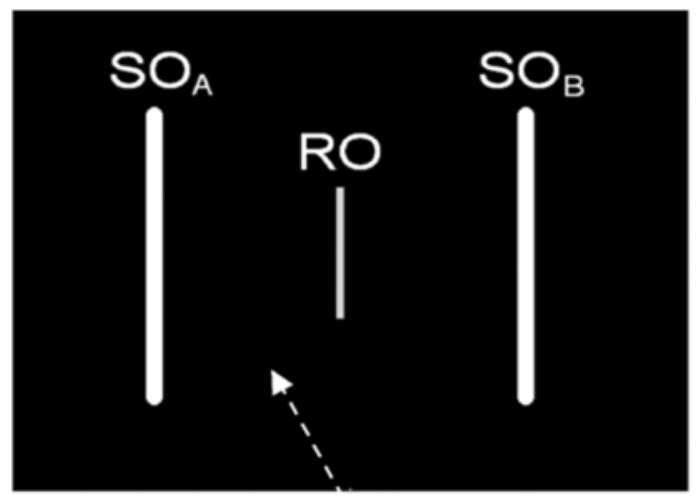

B

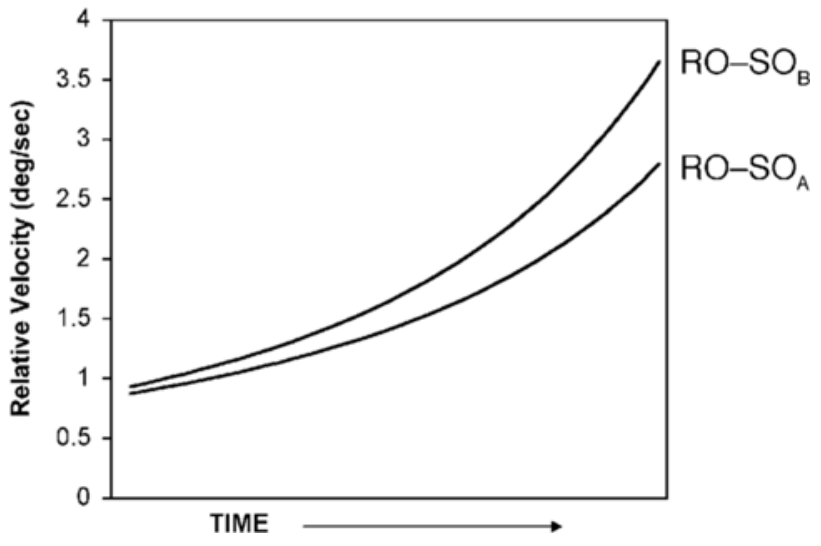

Figure 3. (A) A combined schematic representation of separate two-object displays with the second object (SO) in front of the reference object (RO; $\mathrm{SO}_{\mathrm{A}}$ to the left of $\mathrm{RO}$ and $\mathrm{SO}_{\mathrm{B}}$ to the right). A path between $\mathrm{SO}_{\mathrm{A}}$ and $\mathrm{RO}$ is shown. (B) Velocity-time graphs for the relative motion between objects making up the two pairs in the configuration (i.e., $\mathrm{RO}_{-} \mathrm{SO}_{\mathrm{A}}$ and $\mathrm{RO}^{-\mathrm{SO}} \mathrm{B}_{\mathrm{B}}$ ). $\mathrm{Al}^{-}$ though both pairs diverge and accelerate (AD), the pair for which the path is between the objects $\left(\mathrm{RO}_{-} \mathrm{SO}_{\mathrm{A}}\right)$ does So less rapidly than that for which the path is outside the far member $\left(\mathrm{RO}-\mathrm{SO}_{\mathrm{B}}\right)$. 
average rate of acceleration for the former, in the example depicted in Figure 3, is $0.679 \mathrm{deg} / \mathrm{sec}^{2}$, whereas that for the latter is $0.479 \mathrm{deg} / \mathrm{sec}^{2}$.

This difference between the two far-RO heuristic (AD) path conditions was potentially a contributing factor to the different heading judgments observed by Best et al. (2002) in near-RO conditions. However, there was another related and confounding difference. In the experiments of Best et al., the lateral position of the SO was randomized within ranges that ensured similar initial separations between RO and SO for the two heuristic paths. This was done to ensure that the participants could not discriminate between paths simply on the basis of the initial lateral separation between the objects. Since the initial lateral separation between the objects was similar but the rate of acceleration during trials was systematically different, the size of the final lateral separation between the two objects was correlated with path condition: Paths between the objects (less rapid increase in velocity) resulted in generally smaller final separations than did paths outside the far object (more rapid increase in velocity). This resulted in another factor possibly contributing to the differences between the two far-RO heuristic conditions. That is, it is conceivable that the distinct patterns of responses did not arise because of the change in velocity in the displays but, rather, because of the size of the lateral separation between the objects at the end of each trial. The aim of Experiment 1 was to identify which of these variables, velocity change or final separation, determined heading judgments in this context.

\section{Method}

The simulations in Experiment 1 lasted 4 sec and ended with the point of view $10 \mathrm{~m}$ from the RO, which was always the far member of the object pair. Simulations yielded relative object-image motion of the kind depicted in Figure 3. The SO was located in a depth plane $4 \mathrm{~m}$ in front of the RO. The simulated paths were of two kinds: (1) outside the far-RO and (2) between the objects. The SO always lay in one of the large areas of retinal flow in front of the RO that give rise to $\mathrm{AD}$ - that is, heuristic heading information (see Figure 1). No conditions were included in which the object pairs underwent invariant relative motion (i.e., DD and CV).

In order to resolve the confound between lateral separation and the differences in $\mathrm{SO}$ acceleration described above, the correlation between final separation and path condition was eliminated. This was achieved by manipulating the lateral position of the SO to produce three final lateral separations between the images of the two objects for both heuristic paths (see Figure 4). For this manipulation, the field of view on the SO side of the far-RO was segmented. In what was termed the small lateral separation condition, the SO was positioned so that, at the end of the trial, the ratio of the size of the region between the objects to that outside the $\mathrm{SO}$ was 1:3. This was true for every condition except the small-separation, path-between, $8^{\circ}$ gaze angle condition, in which the SO was positioned slightly farther from the RO. This was necessary to ensure that the SO lay outside the simulated path of movement. In this condition, the ratio was $1: 2.1$. In the medium lateral separation condition, the SO lay halfway between the RO and the edge of the display at the end of the trials; hence, the ratio was 1:1. In the large lateral separation condition, the SO finished near the edge of the screen, with a ratio of 3:1. In terms of visual angle, these three conditions represent final separations of $7^{\circ}, 14^{\circ}$, and $21^{\circ}$ between the two objects in the displays. For the small-separation, path-between, $8^{\circ}$ gaze angle condition, the separation was $8.9^{\circ}$. The relative velocity of the two objects over the course of the displays from Experiment 1 are shown in the velocity-time graphs in Figure 5.

The participants viewed five presentations of each of 24 displays individually and in random order during a single session. The 24 displays were generated by factorial combination of path region (outside RO or between, both giving rise to heuristic information, AD), lateral separation (small, medium, or large), path side relative to RO (left or right), and gaze angle $\left(4^{\circ}\right.$ and $\left.8^{\circ}\right)$.

\section{Results and Discussion}

Table 1 shows the mean percentages of responses made in each of the three categories (collapsed across left/right path side relative to RO) as a function of lateral separation and path condition. The cells with asterisks in Table 1 represent correct responses (see the analysis below).

Percentages of correct heading judgments (collapsed across left/right path side relative to RO) were analyzed in a within-subjects analysis of variance (ANOVA) for which the factors were path region (outside RO or between), lateral separation (small, medium, or large), and gaze angle $\left(4^{\circ}\right.$ and $\left.8^{\circ}\right)$. A significant main effect for path region $[F(1,9)=5.22, p<.05]$ and a significant two-way interaction between path region and lateral separation $[F(2,18)=9.013, p<.01]$ were revealed .

Mean percent correct scores are plotted as a function of path condition and gaze angle in Figure 6 for the three
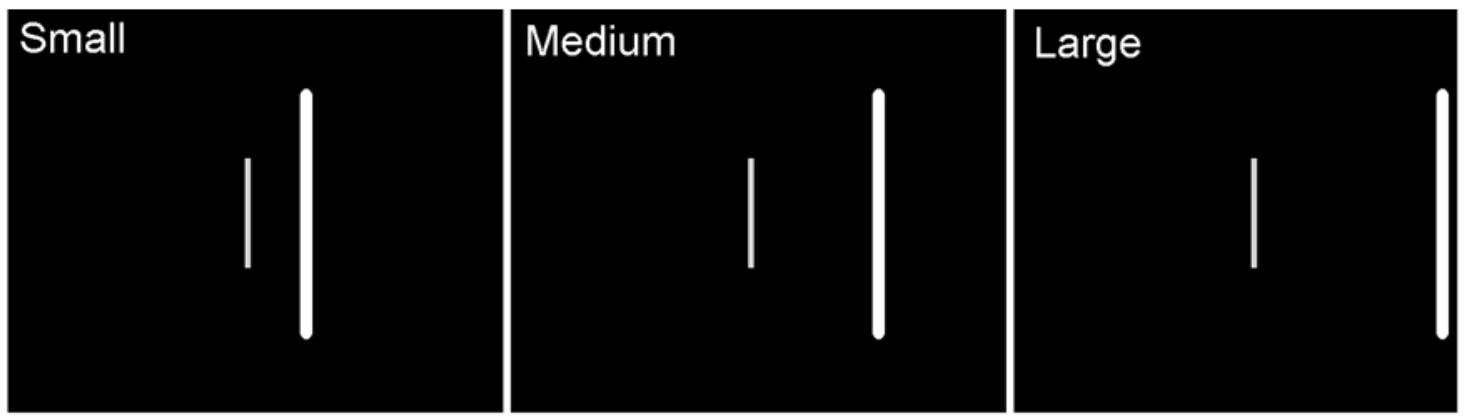

Figure 4. Schematic representations of the three final lateral separation conditions generated in Experiment 1. Displays were manipulated so that the separation between the reference object and the second object at the end of simulated self-movement was small, medium, or large. 


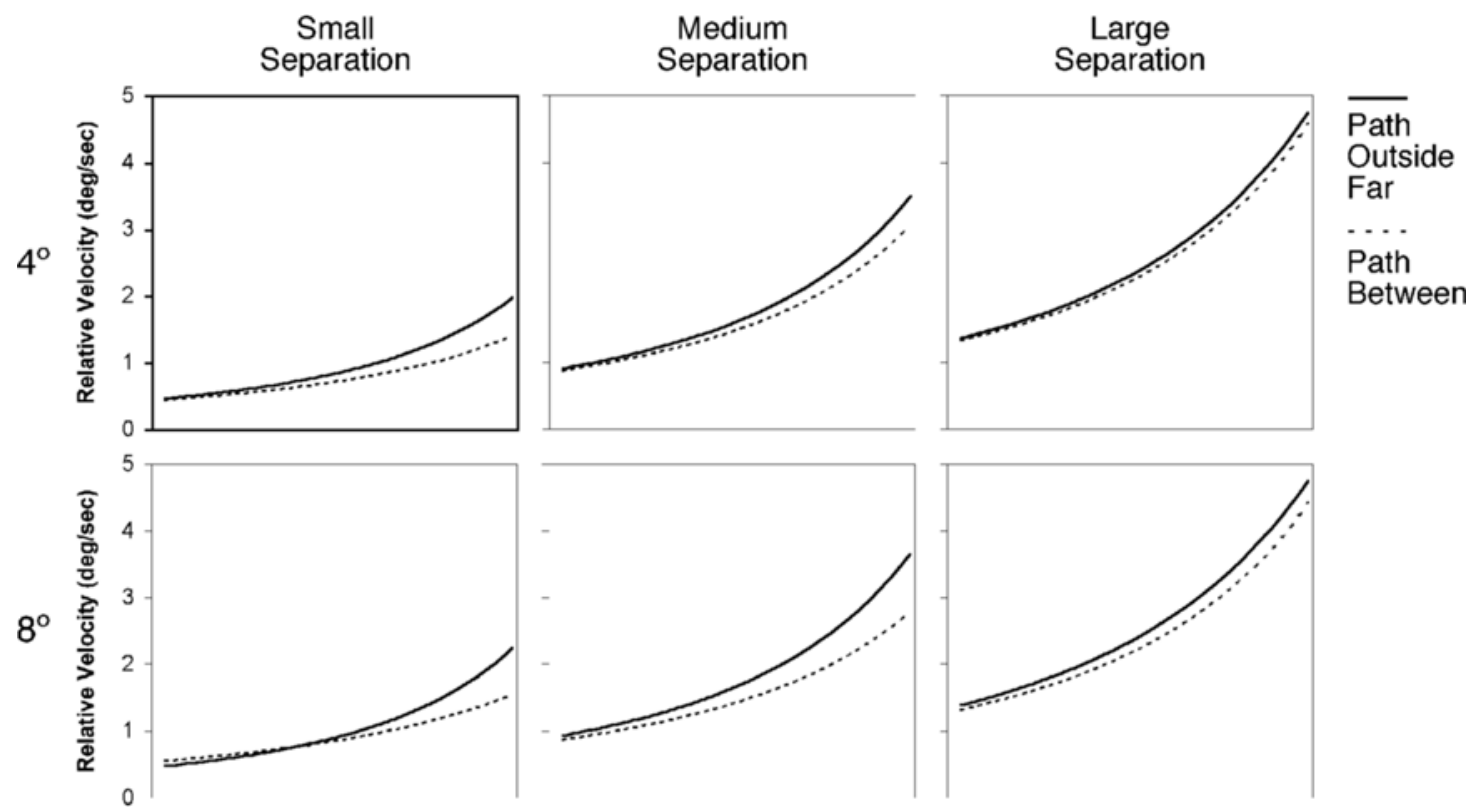

Figure 5. Velocity-time graphs for all the conditions in Experiment 1. All object pairs diverged with accelerating velocity; however, the motion accelerated more rapidly for paths outside the far object.

values of final lateral separation. It can be seen that more correct responses were given for the path-between conditions than for the path-outside-RO conditions. The interaction between path region and separation indicated that there was little difference in heading judgment accuracy between the two path types in the small-separation condition (see the left-hand panel of Figure 6), whereas performance differed between paths in the medium- and the large-separation conditions (see the middle and the right-hand panels of Figure 6, respectively). The difference in the percentage of responses in each category between the two heuristic path conditions is shown in Table 2. It can be seen from Table 2 that there were relatively consistent differences in the patterns of responses obtained in the two heuristic path conditions simulated in Experiment 1. The slightly larger differences in the small-separation condition are reflective of the fact that somewhat fewer judgments were made between the ob-

Table 1

Distribution of Heading Judgments in Experiment 1

\begin{tabular}{llccc}
\hline \multirow{2}{*}{ Separation } & Path & \multicolumn{3}{c}{ Responses } \\
\cline { 3 - 5 } Small & Outside Near & Between & Outside Far \\
& outside-far & 10.5 & $55.5^{*}$ & 34.0 \\
\multirow{2}{*}{ Medium } & 3.5 & 44.0 & $52.5^{*}$ \\
& between & 4.0 & $66.0^{*}$ & 30.0 \\
Large & outside-far & 2.0 & 55.5 & $42.5^{*}$ \\
& between & 3.5 & $63.5^{*}$ & 33.0 \\
& outside-far & 3.0 & 52.0 & $45.0^{*}$ \\
\hline
\end{tabular}

Note-Tabled values are the percentages of responses in each response region, summed across gaze angle, as a function of lateral separation and path condition. * Correct responses corresponding to the values plotted in Figure 6. jects in the small-separation condition. This could be taken as some support for a role of separation. However, the consistency in responses across other conditions suggests that if separation does have an effect, it is small, restricted to very particular situations, and secondary to that of differences in acceleration.

These outcomes confirm those of earlier experiments by Best et al. (2002) and resolve the confound between separation and acceleration. The differences between these path conditions were relatively constant across values of lateral separation, indicating that separation was not likely to be the determining factor for the differences that were observed. These findings, therefore, support the view that, in this context, differences in velocity change are involved in determining heading judgments. However, it is not possible to conclude whether one of the two characterizations of velocity change described above (i.e., ratio or rate) is a more perceptually relevant characterization of the velocity change in these displays, since they were confounded in most conditions.

\section{EXPERIMENT 2}

\section{DD and CV: Two Heading Invariants}

Another consistent finding, in conflict with predictions from the object-based model, that also suggests a role for velocity change is that of the inadequacy of DD as a determinant of heading perception (e.g., Best et al., 2002; Cutting et al., 1998). When object-image pairs undergo DD, they move apart with decelerating velocity. When object-image pairs undergo $\mathrm{CV}$, they move together with accelerating velocity. These differences in direction of relative motion (i.e., converging vs. diverg- 


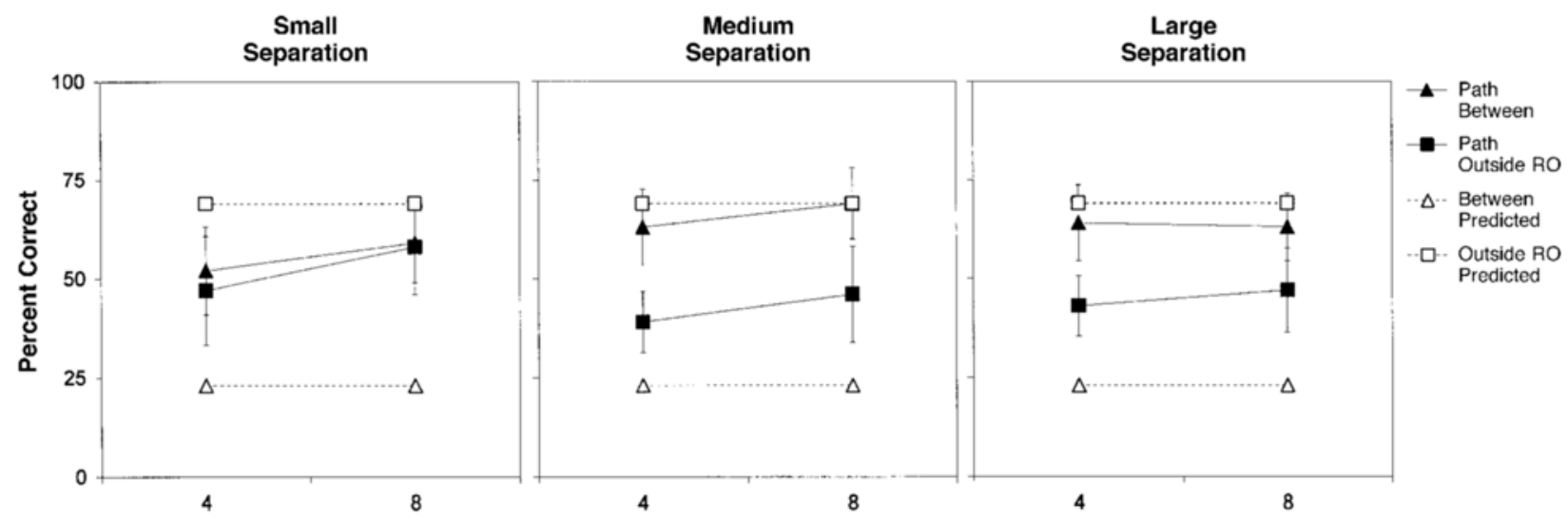

Figure 6. Performance (filled symbols; mean percentages of correct heading judgments, together with $95 \%$ confidence intervals) as a function of path region and gaze angle for Experiment 1. Scores for the small, medium, and large lateral separation conditions are plotted in the left-hand, middle, and right-hand panels, respectively. Predicted performance from the object-based heading model is indicated with unfilled symbols. RO, reference object.

ing) and change in velocity (i.e., acceleration: speeding up vs. slowing down) were accounted for in Wang and Cutting's (1999) object-based model. However, the change in velocity that occurs over time is generally less rapid when object-image pairs undergo DD than when they undergo $\mathrm{CV}$, a difference that was not considered in Wang and Cutting's model.

Cutting et al. (1999) and Cutting and Wang (2000) have demonstrated that DD is a weak determinant of heading judgments, whereas $\mathrm{CV}$ is a strong determinant in both cluttered and sparse environments. Best et al. (2002) have also reported evidence suggesting that the two invariant categories of relative motion (i.e., DD and $\mathrm{CV}$ ) may not be equally effective determinants of heading judgments. When fixation is simulated on a near RO, large areas of the visual field behind the $\mathrm{RO}$ give rise to invariant relative motion (see Figure 1). In the experiments of Best et al., when an SO was placed so that DD was the only invariant present for the duration of the displays, performance was relatively poor. However, when an SO was placed so that $\mathrm{CV}$ was present in the displays, judgments were consistently accurate.

Since it has been shown that the amount of acceleration determines heading judgments in heuristic conditions ( $\mathrm{AD}$; see Experiment 1), it is also possible that this variable plays a role in invariant conditions (DD and

Table 2

Differences in Percentages of Responses in Each Region in Experiment 1 as a Function of Lateral Separation

\begin{tabular}{cccc}
\hline & \multicolumn{3}{c}{ Responses } \\
\cline { 2 - 4 } Separation & Outside Near & Between & Outside Far \\
\hline Small & 7.0 & 11.5 & -18.5 \\
Medium & 2.0 & 10.5 & -12.5 \\
Large & 0.5 & 11.5 & -12.0 \\
\hline
\end{tabular}

Note-Values were obtained by subtracting path outside-RO percentage from path between percentage in Table 1 .
CV). There are two possible explanations for the differences described above: (1) DD does not provide for accurate heading perception under any circumstances, whereas CV does, and (2) the less rapid decelerations in DD conditions lead to poor performance, relative to the more rapid velocity changes in CV conditions. The aim of Experiment 2 was to test these two possibilities.

Conditions were simulated in which the SO was placed in one of the large areas giving rise to invariant information behind a near RO. In these conditions, only one invariant source of relative motion information was present for the duration of the displays (i.e., DD only and $\mathrm{CV}$ only). Comparison between these DD-only and $\mathrm{CV}$-only conditions served to test the first of the possibilities described above. If DD is a poor determinant of heading perception, relative to $\mathrm{CV}$, it can be expected that performance will be more accurate in CV-only conditions than in DD-only conditions. Three types of DD-only conditions were included in Experiment 2. By positioning the SO in different positions within the large area of DD behind a near RO, small, medium, and large decelerations were generated (but see the Method section below). It was predicted that performance would differ between these conditions to the extent that differences in acceleration are related to heading judgments in the presence of DD.

\section{Method}

The apparatus and procedure were the same as those in Experiment 1 . Each participant viewed five presentations of 24 displays in random order. The 24 displays were generated by incomplete factorial combination of path-region/heading information (four kinds of paths outside the RO, giving rise to DD-small, DD-medium, or DD-large velocity change or $\mathrm{CV}$; and paths inside the RO, giving rise to $\mathrm{AD}$ ), path side relative to $\mathrm{RO}$ (left or right), and gaze angle ( $2^{\circ}$ and $4^{\circ}$ for the DD conditions; $2^{\circ}, 4^{\circ}$, and $8^{\circ}$ for the CV and the AD conditions). In Experiment 2, simulated self-movement lasted for $3 \mathrm{sec}$, following which the final frame remained visible for an- 
other $3 \mathrm{sec}$. The two-object configuration in all the displays in Experiment 2 included a near $\mathrm{RO}$ and a far $\mathrm{SO}$. The $\mathrm{SO}$ was positioned in a depth plane $20 \mathrm{~m}$ behind the RO. This configuration was chosen to allow placement of the SO in locations that gave rise to the required kinds of object-image relative motion. Since Best et al. (2002, Experiments 4-6) had shown that the separation in depth between objects in displays of this kind was not a significant factor, it was not expected that the difference between Experiment 1 and Experiment 2 in this regard would influence results.

Three kinds of DD-only conditions, involving DDs of different degrees of deceleration, were generated. The size of DD was defined as the percentage of decrease in velocity over the final $1 \mathrm{sec}$ of the display. This measure corresponds to the velocity ratio described by Schmerler (1976). Each DD magnitude condition was generated by positioning the SO in particular positions along the plane $20 \mathrm{~m}$ behind the RO in the virtual environment. In the $2^{\circ}$ gaze angle condition, decreases in velocity of 1:0.53, 1:0.40, and 1:0.20 over the final second of the displays constituted the small-, medium-, and large-DD conditions, respectively. In the $4^{\circ}$ condition, decreases in velocity of 1:0.57, 1:0.40, and 1:0.20 were included. It should be noted that although these conditions involved different velocity ratios, the rate of deceleration (i.e., in terms of $\mathrm{deg} / \mathrm{sec}^{2}$ ) was similar for all three within each gaze angle condition. In the $2^{\circ}$ condition, rates of change were $0.046,0.05$, and $0.054 \mathrm{deg} / \mathrm{sec}^{2}$ for the small-, medium-, and large-DD conditions, respectively. For the $4^{\circ}$ gaze angle, these rates were also similar, at $0.094,0.104$, and $0.112 \mathrm{deg} / \mathrm{sec}^{2}$. These decelerations are depicted in velocity-time graphs in Figure 7 . It should also be noted that there was a substantial difference in the separations between objects in the two gaze angle conditions. In the $2^{\circ}$ gaze angle condition, the lateral separations between the $\mathrm{RO}$ and the $\mathrm{SO}$ at the beginning of the displays were $5.9^{\circ}, 5.4^{\circ}$, and $4.9^{\circ}$ for the small, medium, and large decelerations, respectively. In the $4^{\circ}$ condition, these values were $13.4^{\circ}, 11.5^{\circ}$, and $10.3^{\circ}$, respectively.

In addition to $\mathrm{DD}$-only conditions, $\mathrm{CV}$-only and $\mathrm{AD}$-only conditions were also simulated. In these conditions, the lateral position of the SO in the virtual environment and, therefore, the angular separation between the images of the $\mathrm{SO}$ and the $\mathrm{RO}$ at the beginning of the trials were randomized within ranges that ensured that the required kind of relative motion took place. The initial angular separation between the object images ranged between $1.1^{\circ}$ and $5.9^{\circ}$ for
CV-only conditions (i.e., paths outside the RO) and between $4.9^{\circ}$ and $10.1^{\circ}$ for $\mathrm{AD}$-only conditions (i.e., paths inside the $\mathrm{RO}$ ). $\mathrm{AD}$ (i.e., heuristic) conditions were generated primarily so that the correct response was not outside the near object in all the conditions.

It was originally intended to include three levels of gaze angle for each condition. Although this proved possible for two conditions, $\mathrm{AD}$ - and CV-only, it did not for $\mathrm{DD}$-only conditions. The requisite SO positions meant that for the $8^{\circ} \mathrm{DD}$-only conditions, the SO was so peripheral as to be outside the field of view presented in the display. The experimental design therefore included three levels of gaze angle $\left(2^{\circ}, 4^{\circ}\right.$, and $\left.8^{\circ}\right)$ for $\mathrm{AD}$ heuristic conditions and CV-only invariant conditions, but only two levels $\left(2^{\circ}\right.$ and $\left.4^{\circ}\right)$ for DD-only conditions. In order to achieve a complete factorial design in the ANOVA on invariant conditions (see the Results and Discussion section below), the $\mathrm{CV}$-only, $8^{\circ}$ gaze angle condition was excluded from analysis. Nevertheless, the data from this condition are plotted in all the graphs and are included in all the tables relating to the experiment.

\section{Results and Discussion}

Table 3 shows the mean percentages of responses made in each of the three categories (collapsed across left/right path side relative to RO) as a function of path condition in Experiment 2. The asterisked figures in Table 3 represent correct responses (see the analysis below). Percentages of correct heading judgments were analyzed by means of two within-subjects ANOVAs-one for the data from heuristic conditions and the other for those from invariant conditions. These conditions were analyzed separately for the following reasons: (1) Comparisons between invariant and heuristic conditions were not central to the aims of the experiment, and (2) the fundamental difference between heuristics and invariants in perceptual theory makes such comparison something of a "metatheoretical oxymoron" (Cutting \& Wang, 2000, p. 1157).

Each of the two ANOVAs in Experiment 2 was tested at the .025 alpha level, a strategy that maintains the same
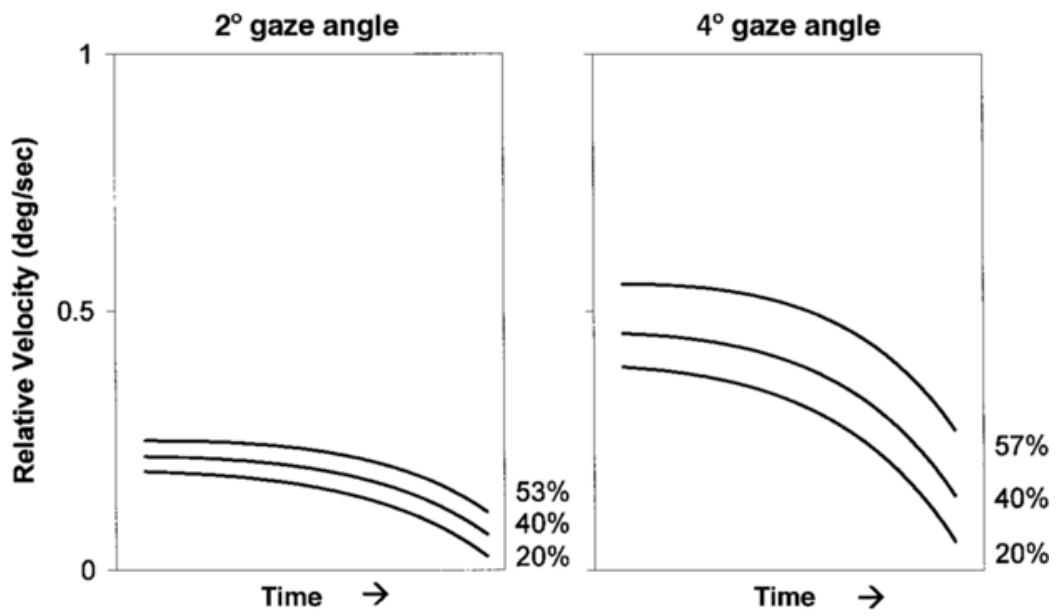

Figure 7. Velocity-time graphs representing the relative motion of objects in the decelerating divergence conditions of Experiment 2. Although the velocity ratio is different for each condition within gaze angle, the rate of deceleration is similar. 
Table 3

Distribution of Heading Judgments in Experiment 2

\begin{tabular}{lccc}
\hline & \multicolumn{3}{c}{ Responses } \\
\cline { 2 - 4 } Path/Information Category & Outside Near & Between & Outside Far \\
\hline Heuristic (AD) & $23.3^{*}$ & $73.7^{*}$ & 3.0 \\
Small deceleration (DD) & $57.5^{*}$ & 42.5 & 0.0 \\
Medium deceleration (DD) & $58.5^{*}$ & 41.0 & 0.5 \\
Large deceleration (DD) & $60.0^{*}$ & 38.5 & 1.5 \\
Convergence (CV) & $81.7^{*}$ & 6.7 & 11.7 \\
\hline
\end{tabular}

Note-Tabled values are the percentage of responses in each response region, summed across gaze angle. * Correct responses corresponding to the values plotted in Figure 7

experiment-wise error rate of .05 for Experiments 1 and 2 . The ANOVA on invariant conditions included the three factors of information type (small, medium, or large DD or CV) and gaze angle $\left(2^{\circ}\right.$ and $\left.4^{\circ}\right)$. The ANOVA on heuristic conditions included only the gaze angle factor $\left(2^{\circ}, 4^{\circ}\right.$, and $\left.8^{\circ}\right)$. In the ANOVA on invariant conditions, a significant main effect was found for information type $[F(2,18)=6.64, p<.01]$. No other main effects or interactions proved significant. In the ANOVA conducted on heuristic conditions, no significant effect of gaze angle was revealed. Mean scores are plotted as a function of path/heading information condition and gaze angle in Figure 8 for conditions giving rise to invariant heading information (path outside RO; left-hand panel) and conditions giving rise to heuristic information (path inside RO; right-hand panel). The separate curves in the left panel represent the three kinds of DD-only conditions, in addition to the CV-only condition.

Performance was closer to predictions in all the $\mathrm{CV}$-only invariant conditions than in any of the DD-only conditions (see the left-hand panel). Performance was also close to predictions in the AD conditions (see the right-hand panel). These results imply that the invariant $\mathrm{CV}$ is an effective determinant of heading perception, whereas DD is not, despite its status as an invariant. It can be seen from Table 3 that performance was quite different in the presence of the two kinds of diverging motion, $\mathrm{AD}$ and $\mathrm{DD}$. This indicates that the poor performance in DD conditions was not likely to be due to an inability on the part of the participants to discriminate between $\mathrm{AD}$ and $\mathrm{DD}$. It appears that the participants did discriminate between diverging motion that sped up (AD) and that which slowed down (DD). Although the difference between these conditions was apparent to the participants, they did not make effective use of the invariant specification of heading afforded by DD. Inspection of Figure 8 shows that there was no clear effect of velocity change in DD conditions in terms of Schmerler's (1976) velocity ratio. This conflicts with the findings of Experiment 1, in which it was found that velocity change was an important determinant of heading judgments. However, within gaze angles, DD conditions with different velocity ratios were similar in terms of rate of acceleration. Therefore, it remains possible that velocity change is an important determinant of perceived heading but that the more perceptually relevant characterization of velocity change is the rate of acceleration as described above. This issue is considered in more detail in the General Discussion section below.

\section{GENERAL DISCUSSION}

Experiments 1 and 2 led to the resolution of two issues associated with the object-based model of heading perception. First, the influence of lateral separation and velocity change in heuristic heading judgments was examined. Although heuristic pairs that are widely sepa-
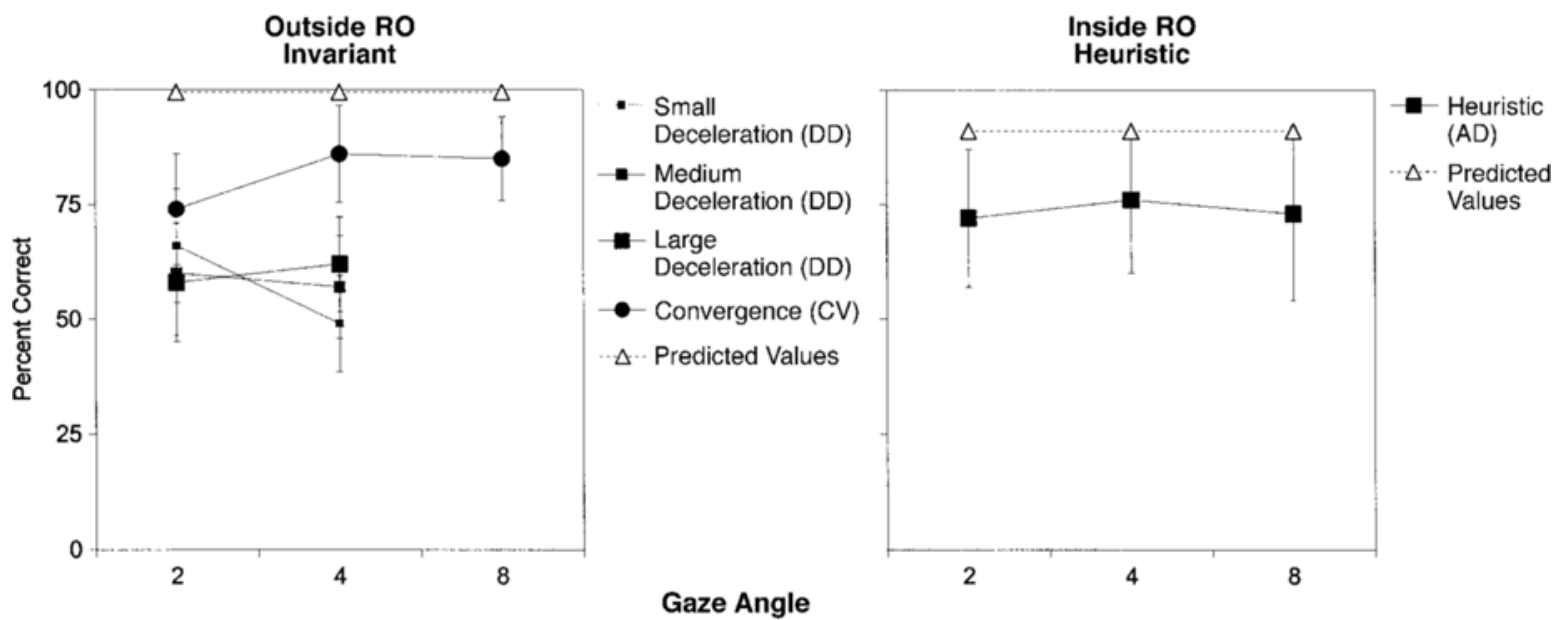

Figure 8. Performance (filled symbols; mean percentages of correct heading judgments, together with $95 \%$ confidence intervals) as a function of path-region/heading information and gaze angle for Experiment 2 . Scores for conditions giving rise to invariant heading information are shown in the left panel; those for conditions giving rise to heuristic heading information are shown in the right panel. Predicted performance from the object-based heading model is indicated with unfilled symbols. RO, reference object; $D D$, decelerating divergence; $A D$, accelerating divergence. 
rated necessarily accelerate apart at higher velocity, objects of a given separation accelerate more rapidly and travel at greater velocities for paths outside the RO than for paths between the objects. Across lateral separations, the percentage of between and outside-far heading judgments was similar within each of the two heuristic (AD) path conditions. However, the pattern of responses differed between these two path conditions: There were generally more between responses for the objects in the path-between conditions than in the path-outside-RO conditions. There was one condition in which more outside far judgments were made than between judgments. This was the path-outside-RO, small lateral separation condition. This outcome indicates that lateral separation of objects could have played a role in heading perception from $\mathrm{AD}$. However, given the results from the other conditions in Experiment 1, it appears that the effect of lateral separation may be merely that responses are less likely to be made in a given response category when the region of the visual field corresponding to that category is quite small. This lateral separation effect was the opposite of that which would be expected if separation was the determinant of the difference observed in the experiments conducted by Best et al. (2002). That is, in the experiments by Best et al., more between responses were made in path-between conditions, a situation in which the region between the two objects was relatively small.

Importantly, the different patterns of responses observed in the two kinds of heuristic path conditions in previous experiments were observed across all three values of lateral separation in Experiment 1. For object pairs of given lateral separation undergoing $\mathrm{AD}$, the less rapid the acceleration, the more likely it is that the path will be judged to be between the objects. Therefore, contrary to the predictions of Wang and Cutting's (1999) objectbased model, one pair of objects undergoing AD should not be considered perceptually equivalent to all others. Heading is reliably perceived as inside the near member of object pairs undergoing AD. However, details of motion, such as rate of acceleration or velocity ratio, are necessary, in addition to the relative motion category, to account for where inside the near object the direction of heading is judged to be: between the objects or outside the far object.

Second, in terms of the object-based invariants DD and $\mathrm{CV}$, it was found that DD is a weak determinant of heading perception, relative to CV. This was independent of the magnitude of the velocity ratio in DD displays. Although invariant conditions involving DD gave rise to performance that was generally in the expected direction, performance was far from being in accord with predictions. Invariant conditions containing only $\mathrm{CV}$, however, gave rise to performance that was close to predictions. Multiple invariants were not necessary for accurate performance in invariant conditions. Although small-, medium-, and large-DD conditions differed in terms of velocity ratios, they were similar within gaze angles in terms of rate of acceleration. Therefore, it remains possible that velocity change is an important determinant of perceived heading in this context but that the more perceptually relevant characterization of velocity change is rate of acceleration, rather than velocity ratio.

In support of this idea, some evidence for a role for rate of acceleration in heading perception is provided by examination of this variable in relation to percentage of correct responses across all invariant conditions in Experiment 2. In Experiment 2, the two invariants (i.e., DD and $\mathrm{CV})$ were generated in separate conditions. Six conditions contained DD (small, medium, and large DD, defined in terms of velocity ratios, each with $2^{\circ}$ and $4^{\circ}$ gaze angles) and three conditions contained $\mathrm{CV}\left(2^{\circ}, 4^{\circ}\right.$, and $8^{\circ}$ gaze angles). The rates of acceleration in conditions involving DD were $0.046,0.050,0.054,0.094,0.105$, and $0.112 \mathrm{deg} / \mathrm{sec}^{2}$. In conditions containing $\mathrm{CV}$, the accelerations were generally higher, with means across random initial separation ranges of $0.085,0.174$, and $0.349 \mathrm{deg} / \mathrm{sec}^{2}$. These rates of acceleration, along with the percentage of responses placed correctly on the outside of the near object, in each of these conditions are shown in Figure 9.

Two points of interest emerge from these data. First, although most responses were judged to be in the expected region in all of these conditions, higher rates of acceleration were associated with elevated percentages of outside the near object responses. The mean rate of acceleration for DD conditions was $0.076 \mathrm{deg} / \mathrm{sec}^{2}$, whereas that for $\mathrm{CV}$ was $0.203 \mathrm{deg} / \mathrm{sec}^{2}$; the mean percentages of outside the near object responses corresponding to these conditions were $58.6 \%$ and $81.7 \%$, respectively. Second, there was one CV condition in which acceleration was of similar magnitude to that in the DD conditions. In terms of $95 \%$ confidence intervals, performance in this condition overlapped considerably with that from the DD conditions, whereas performance in the $\mathrm{CV}$ conditions with greater rates of acceleration did not. In fact, the confidence interval for the $2^{\circ} \mathrm{CV}$ condition contained the means of three of the DD conditions. The means of another two DD conditions fell just outside that range (see Figure 9). The confidence intervals for the $\mathrm{CV}$ conditions that involved relatively large changes in velocity ( $4^{\circ}$ and $8^{\circ}$ gaze angles) contained none of the DD condition means. Although it is not possible to infer a causal relationship from these data, the covariation of rate of acceleration and percentage of responses placed correctly outside the near object suggests that rate of acceleration plays a role in the determination of heading perception provided by invariant relative motion.

The pattern of responses reported here is at least consistent with the idea that the participants were sensitive to differences in acceleration underlying the categories identified by Wang and Cutting (1999). This raises the question of why such information might be used. An answer may be found by considering the added specification of the direction of heading that is available from differences in acceleration - in particular, with the heuristic, AD. Differences in acceleration provide a means by which observers can discriminate between paths that lead between a pair of objects and those that lead to the 


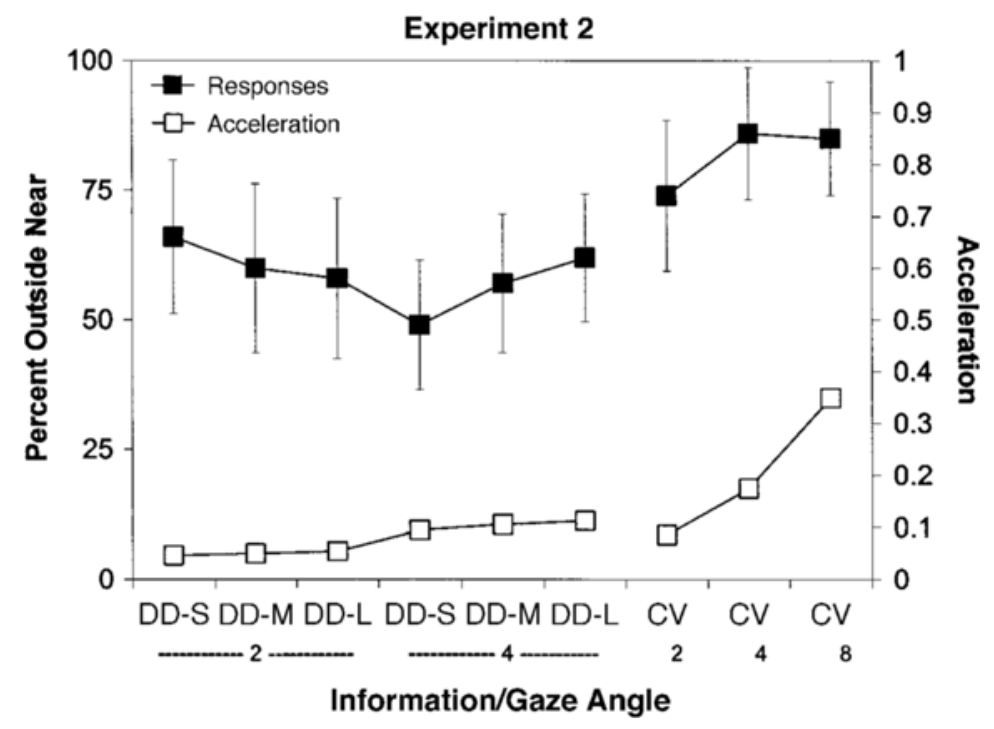

Figure 9. Rate of negative acceleration $\left(\mathrm{deg} / \mathrm{sec}^{2}\right)$ and percentage of outside the near object responses (as predicted) in invariant conditions in Experiment 2. Responses are plotted on the left vertical axis, whereas rate of acceleration is plotted on the right vertical axis. Conditions with relatively low rates of acceleration resulted in fewer outside the near object responses. Conversely, conditions with higher rates of acceleration resulted in more such responses. DD, decelerating divergence; $\mathrm{CV}$, convergence; $\mathrm{S}$, small; $\mathrm{M}$, medium; L, large.

outside of the farther member. For objects of a given separation, faster acceleration takes place when one is heading toward the outside of the far object than when one is heading between the objects. If AD is considered a single source of information, as under the model of Wang and Cutting, no such discrimination is possible.

We might also consider what implications these findings might have for the object-based model itself. That is, what would a model of heading perception based on such information look like? A simple modification of the object-based model that could be made to relate it more closely to the variable of relative retinal image acceleration would be to consider acceleration as positive or negative. ${ }^{3}$ $\mathrm{AD}$ represents an instance of positive acceleration with varying rates of change that appear to be linked to changes in heading judgments. DD and CV represent, respectively, relatively slow velocity change and relatively fast velocity change instances of negative acceleration. When a pair of object images undergoes positive acceleration (AD), one is almost certainly (i.e., with around $90 \%$ certainty) heading to the inside of the nearer object (i.e., either between or outside the far object). Furthermore, the amount of acceleration relates to where inside that object the path of movement is leading: between the objects (smaller positive acceleration) or outside the farther object (larger positive acceleration). When a pair of object images undergoes negative acceleration (DD or CV), one is heading to the outside of the nearer object. This excludes the necessity to consider the farther object of the pair. Passing to the inside or outside of a nearby object, then, could be seen to become a process of regulating retinal image acceleration. That is to say, the ability to regulate retinal image acceleration-for example, keeping such acceleration positive or negative-during locomotion $i s$ the ability to pass to the inside or the outside of the objects that furnish the environment. In this way, retinal image acceleration could be considered similar to the kind of ecological information that Gibson sought (Reed, 1996). Although this would appear to be a relatively parsimonious way to account for the use of retinal image accelerations in heading perception, it is important to note that these suggestions are speculative and have yet to be the subject of empirical scrutiny.

In conclusion, although the experiments reported here provide some support for the predictions of the objectbased model (Wang \& Cutting, 1999), some consistent departures from expected outcomes (Best et al., 2002) have been confirmed. The data suggest a role for differences in velocity change (acceleration) in determining perceived heading in a minimalist context. This is an interesting and potentially important finding, since changes in velocity are relatively difficult to detect (Schmerler, 1976). Nevertheless, it is clear that changes in velocity did play a role in this context, at least with regard to the AD heuristic and, possibly, the DD and CV invariants. Therefore, although the direction of heading relative to objects in the environment can be perceived with a degree of accuracy from the retinal image relative motion of just two objects, the way in which this is achieved may be different from that described in Wang and Cutting's model. 


\section{REFERENCES}

Best, C. J., CRAssini, B., \& Day, R. H. (2002). The roles of static depth information and object-image relative motion in perception of heading. Journal of Experimental Psychology: Human Perception \& Performance, 28, 884-901.

Beusmans, J. M. H. (1998). Perceived object shape affects the perceived direction of self-movement. Perception, 27, 1079-1085.

CutTing, J. E. (1986). Perception with an eye for motion. Cambridge, MA: MIT Press.

CutTing, J. E. (1996). Wayfinding from multiple sources of local information in retinal flow. Journal of Experimental Psychology: Human Perception \& Performance, 22, 1299-1313.

Cutting, J. E., Alliprandini, P. M. Z., Creutz, E. A., \& Wang, R. F. (1998). Heading judgments and information seeking: The purposive behavior of eye movements [Abstract]. Investigative Ophthalmology \& Visual Science, 39(Suppl.), 892.

Cutting, J. E., Springer, K., Braren, P. A., \& Johnson, S. H. (1992). Wayfinding on foot from information in retinal, not optical, flow. Journal of Experimental Psychology: General, 121, 41-72.

Cutting, J. E., Vishton, P. M., Flückiger, M., Baumberger, B., \& Gerndt, J. D. (1997). Heading and path information from retinal flow in naturalistic environments. Perception \& Psychophysics, 59, 426-441.

Cutting, J. E., \& WAng, R. F. (2000). Heading judgments in minimal environments: The value of a heuristic when invariants are rare. Perception \& Psychophysics, 62, 1146-1159.

Cutting, J. E., Wang, R. F., Flǘckiger, M., \& Baumberger, B. (1999). Human heading judgments and object-based motion information. Vision Research, 39, 1079-1105.

GiBson, J. J. (1950). Perception of the visual world. Boston: Houghton Mifflin.

REED, E. S. (1996). Encountering the world: Toward an ecological psychology. New York: Oxford University Press.

Regan, D., \& Beverley, K. I. (1982). How do we avoid confounding the direction we are looking and the direction we are moving? Science, 215, 194-196.

Schmerler, J. (1976). The visual perception of accelerated motion. Perception, 5, 167-185.
Vishton, P. M., \& Cutting, J. E. (1995). Wayfinding, displacements and mental maps: Velocity fields are not typically used to determine one's aimpoint. Journal of Experimental Psychology: Human Perception \& Performance, 21, 978-995.

Vishton, P. M., Nijhawan, R., \& Cutting, J. E. (1994). Moving observers utilise static depth cues in determining their direction of motion [Abstract]. Investigative Ophthalmology \& Visual Science, 35(Suppl.), 2000.

Wallach, H., Stanton, L., \& Becker, D. (1974). The compensation for movement-produced changes of object orientation. Perception \& Psychophysics, 15, 339-343.

WANG, R. F., \& CutTing, J. E. (1999). Where we go with a little, good information. Psychological Science, 10, 71-75.

\section{NOTES}

1. In this article, as in the object-based model under investigation, we will use the term heading to refer to the region of the visual field, defined relative to objects in the environment, toward which the perceived direction of movement will eventually lead the observer.

2. The use of the label object-based approach is meant only to highlight a difference in emphasis between this and other models of perceived heading. This model focuses explicitly on the relative motions of objects in the visual field and yields predicted directions of perceived heading relative to those objects.

3. In formal usage, positive acceleration involves both motion away with increasing velocity as well as motion toward with decreasing velocity, whereas negative acceleration involves both motion away with decreasing velocity and motion toward with increasing velocity. Only three of these four combinations of direction of motion and change in velocity are present in retinal flow as analyzed by Wang and Cutting (1999; see the introduction above). A simple rule of thumb for determining the direction of acceleration is that an object that is slowing down will have an acceleration in the direction opposite its motion.

(Manuscript received April 22, 2002; revision accepted for publication April 23, 2003.) 